\title{
Personalized Medicine (Aim, Role \& Benefits)
}

\section{Bassam Abdul Rasool Hassan*}

Clinical pharmacy discipline, School of Pharmaceutical Sciences, University of Sains Malaysia, 11800, Minden, Penang, Malaysia

\section{General Introduction}

Personalized medicine term refer to the designing of medical treatment based on individual characteristics of each patient i.e., it is the process of tailoring treatment for each patient. The main factor that plays a critical role in personalizing medicine is the genetic information. Therefore, utilizing of genetic information will mainly lead to increases in the benefits obtain from using a medical treatment and in the same time will reduce the possibility of side effects that might associated with it use.

Moreover, personalized medicine refers to or aims to forecast susceptibility of each individual to critical medical problems, by this way it works on to specify steps that may help in preventing or reducing the probability of individual sufferance from a disease(s) and/ or treatment side effect(s).

\section{Essential Factors that Play Significant Role in Making Personalized Medicine Fully Beneficial}

These factors are the following:

1 - Medical report for each patient must be available.

2 - Full genomic data for each individual involved in the clinical research must be available.
3 - All the electronic tools that support physicians in taking the correct decision must be freely accessible.

4 - A specific and suitable health plan must be available.

5 - All the personal clinical information must be available for clinical researcher (s).

\section{Role and Benefits of Personalized Medicine in Near} Future

This field plays an effective role in changing our way of thinking relating diagnosis and treatment of medical problem (s). This will be done through its significant way in improving and developing our theories, skills, and technologies used in both the clinical and the supportive and palliative care fields. Therefore, all of the abovementioned points will lead to a significant improvement of disease diagnoses and treatment (i.e., medication administer for each patient characterized by an accurate mechanism of action, high level of effectiveness and efficiency and low or restricted side effects).

\section{Conclusion}

Therefore it is an obligate matter for all the open access journals to encourage researchers and clinicians to work hard in order to clarify the main benefits and importance of personalized medicine.
*Corresponding author: Bassam Abdul Rasool Hassan, Clinical Pharmacy discipline, School of Pharmaceutical Sciences, University of Sains Malaysia, 11800 Minden, Penang, Malaysia, Tel: +60164230950; E-mail: bassamsunny@yahoo.com

Received January 07, 2014; Accepted January 10, 2014; Published January 14,2014

Citation: Hassan BAR (2014) Personalized Medicine (Aim, Role \& Benefits) . Nat Prod Chem Res 2:e105. doi:10.4172/2329-6836.1000e105

Copyright: $\odot 2014$ Hassan BAR. This is an open-access article distributed under the terms of the Creative Commons Attribution License, which permits unrestricted use, distribution, and reproduction in any medium, provided the original author and source are credited. 